J. Beerten, S. Cole, and R. Belmans, "Generalized steady-state VSC MTDC model for sequential AC/DC power flow algorithms," IEEE Trans. Power Syst., vol. 27, no. 2, pp. 821-829, May. 2012.

Digital Object Identifier: 10.1109/TPWRS.2011.2177867

URL:

http://ieeexplore.ieee.org/xpl/abstractAuthors.jsp?arnumber=6123184

(C) 2012 IEEE. Personal use of this material is permitted. Permission from IEEE must be obtained for all other users, including reprinting/ republishing this material for advertising or promotional purposes, creating new collective works for resale or redistribution to servers or lists, or reuse of any copyrighted components of this work in other works. 


\title{
Generalized Steady-State VSC MTDC Model for Sequential AC/DC Power Flow Algorithms
}

\author{
Jef Beerten, Student Member, IEEE, Stijn Cole, Member, IEEE, and Ronnie Belmans, Fellow, IEEE
}

\begin{abstract}
In this paper, a steady-state Multi-terminal Voltage Source Converter High Voltage Direct Current (VSC MTDC) model is introduced. The proposed approach is extended to include multiple AC and DC grids with arbitrary topologies. The DC grids can thereby interconnect arbitrary buses in one or more non-synchronized AC systems. The converter equations are derived in their most general format and correctly define all setpoints with respect to the system bus instead of the converter or filter bus, which is often done to simplify calculations. The paper introduces a mathematical model to include the converter limits and discusses how the equations change when a transformerless operation is considered or when the converter filter is omitted. An AC/VSC MTDC power flow is implemented using MATPOWER to show the validity of the generalized power flow model.
\end{abstract}

Index Terms-HVDC transmission, HVDC converters, load flow analysis, VSC HVDC.

\section{INTRODUCTION}

$\mathbf{T}$ HE ever growing need for transmission capacity in current day power systems has led to an increased interest in transmission based on Voltage Source Converter High Voltage Direct Current (VSC HVDC) technology. One of the advantages of the VSC technology over conventional Current Source Commutated (CSC) HVDC is the relatively straightforward extension to Multi-Terminal (MTDC) configurations. The VSC HVDC offers significant advantages over traditional AC grid reinforcements and an operation in a MTDC grid could facilitate the integration of renewable energy sources in the future. Recently, plans have even been suggested to build offshore grids based on DC technology or a DC supergrid to connect the growing share of renewable energy sources [1], [2].

One of the outstanding research issues is the steady-state behavior of these integrated AC/DC systems. In the past, numerous efforts have been spent on the joint solution of integrated AC/DC systems based on the traditional CSC technology [3]-[7]. Generally, the solution methods presented for CSC HVDC systems can be subdivided in unified and sequential methods. In unified methods, the AC and DC system equations are solved together [6], whereas in the sequential method, the $\mathrm{AC}$ and $\mathrm{DC}$ system equations are solved sequentially

Jef Beerten is funded by a research grant from the Research Foundation Flanders (FWO).

Jef Beerten and Ronnie Belmans are with the Department of Electrical Engineering (ESAT), Division ELECTA, Katholieke Universiteit Leuven, Kasteelpark Arenberg 10, bus 2445, 3001 Leuven-Heverlee, Belgium. (e-mail: jef.beerten@esat.kuleuven.be, ronnie.belmans@esat.kuleuven.be)

Stijn Cole is with the Power System Consulting group of Tractebel Engineering, Arianelaan 7, 1200 Brussels, Belgium (e-mail: stijn.cole@gdfsuez.com).
[7]. The biggest advantage of the sequential methods over their unified counterparts is that the sequential methods can be implemented relatively easily as an extension to existing AC power flow programs, whereas a unified implementation requires an alteration of existing $\mathrm{AC}$ power flow algorithms.

A lot of research has been conducted on the operation of CSC HVDC in a multi-terminal set-up, but the inherent technical specifics of the CSC technology make a multiterminal operation impractical if more than three converters are involved, thus impeding an application of the CSC technology in large scale, meshed DC grids. In a parallel operation, a power reversal is complex as it requires mechanical switching. A different voltage to ground at the different converters results in a complex insulation and grounding scheme in a series operation, hampering an extension of the scheme. VSC HVDC has much better prospects for an operation in a MTDC system than CSC HVDC technology: The DC side of the converter behaves as a current source, which makes a power reversal at any terminal a straight-forward control set-point change.

The general distinction between unified and sequential methods is also applicable to integrated AC/VSC MTDC power flow algorithms, but due to the technical specifics of the VSC, the power flow models of CSC and VSC MTDC systems differ. Whereas the past research has been focussing on the power-flow solutions for CSC MTDC systems, a general approach for VSC HVDC systems is missing. Most VSC HVDC power flow models available in literature or embedded in commercial software are limited to two-terminal VSC HVDC systems or are not general enough, which can impede their extension to a general VSC MTDC model [8]. In [9], [10], the analysis is limited to a two-terminal VSC HVDC system. In [11], converter losses are neglected, DC variables are not accessible and the power flow set-points are defined at the converter bus instead of the system bus. This simplifies the calculations, but is not in accordance with current practice. In [12], losses are included but the VSC is only modeled to a limited extent while converter limits are not included.

The main contribution of this paper is the development of a detailed, general steady-state VSC MTDC model for a sequential AC/DC power flow algorithm. The method elaborates further on the general algorithm presented in [13], now including a representation of converter transformers, filters and operation limits as a part of the converter model. Additionally, the model can represent multiple DC grids interconnecting different buses within one AC system or connecting buses from various AC systems. The paper also discusses the modifications to be made when a transformer- or filterless operation 


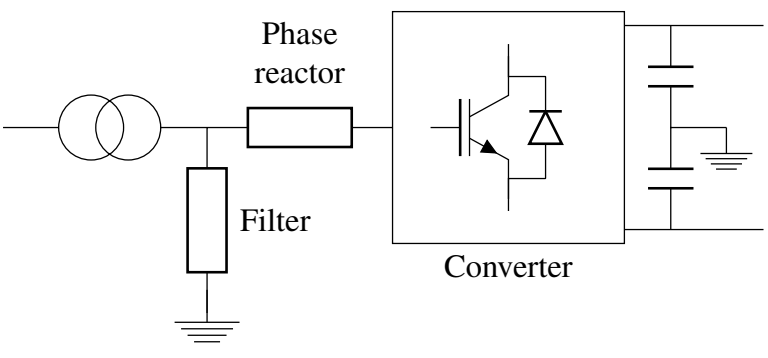

Fig. 1. VSC HVDC converter station.

is considered. Section II discusses the power flow model of the converter and that of the DC grid. The sequential AC/DC power flow implementation is discussed in Section III. Finally, Section IV discusses simulation results by combining the proposed methodology with the open source Matlab toolbox, MATPOWER [14].

\section{POWER FLOW MODELING}

\section{A. Converter model}

In its most general format, a VSC HVDC converter (Fig. 1) can be represented by a controllable voltage source $\underline{U}_{c}=$ $U_{c} \angle \delta_{c}$ behind the phase reactor with a complex impedance $\underline{Z}_{c}=R_{c}+\jmath X_{c}$. As shown in Fig. 2, the phase reactor is connected to the susceptance $B_{f}$ which forms a part of the low pass filter. The filter bus is connected to the $\mathrm{AC}$ grid through a transformer, represented by its impedance $\underline{Z}_{t f}=R_{t f}+\jmath X_{t f}$. The equations for the power flowing to the $\mathrm{AC}$ network can thus be written as

$P_{s}=-U_{s}^{2} G_{t f}+U_{s} U_{f}\left[G_{t f} \cos \left(\delta_{s}-\delta_{f}\right)+B_{t f} \sin \left(\delta_{s}-\delta_{f}\right)\right]$,

$Q_{s}=U_{s}^{2} B_{t f}+U_{s} U_{f}\left[G_{t f} \sin \left(\delta_{s}-\delta_{f}\right)-B_{t f} \cos \left(\delta_{s}-\delta_{f}\right)\right]$,

with $\underline{U}_{s}=U_{s} \angle \delta_{s}$ and $\underline{U}_{f}=U_{f} \angle \delta_{f}$ respectively the complex grid side and filter bus voltage. The equations at the converter side can be written as

$$
\begin{aligned}
& P_{c}=U_{c}^{2} G_{c}-U_{f} U_{c}\left[G_{c} \cos \left(\delta_{f}-\delta_{c}\right)-B_{c} \sin \left(\delta_{f}-\delta_{c}\right)\right], \\
& Q_{c}=-U_{c}^{2} B_{c}+U_{f} U_{c}\left[G_{c} \sin \left(\delta_{f}-\delta_{c}\right)+B_{c} \cos \left(\delta_{f}-\delta_{c}\right)\right],
\end{aligned}
$$

Assuming the AC filters to be lossless, the filter power reduces to its reactive part

$$
Q_{f}=-U_{f}^{2} B_{f},
$$

while the expressions for the filter side complex power flowing through the transformer are written as

$P_{s f}=U_{f}^{2} G_{t f}-U_{f} U_{s}\left[G_{t f} \cos \left(\delta_{s}-\delta_{f}\right)-B_{t f} \sin \left(\delta_{s}-\delta_{f}\right)\right],(6)$

$Q_{s f}=-U_{f}^{2} B_{t f}+U_{f} U_{s}\left[G_{t f} \sin \left(\delta_{s}-\delta_{f}\right)+B_{t f} \cos \left(\delta_{s}-\delta_{f}\right)\right],(7)$

and those flowing through the phase reactor side are

$$
\begin{aligned}
& P_{c f}=-U_{f}^{2} G_{c}+U_{f} U_{c}\left[G_{c} \cos \left(\delta_{f}-\delta_{c}\right)+B_{c} \sin \left(\delta_{f}-\delta_{c}\right)\right], \\
& Q_{c f}=U_{f}^{2} B_{c}+U_{f} U_{c}\left[G_{c} \sin \left(\delta_{f}-\delta_{c}\right)-B_{c} \cos \left(\delta_{f}-\delta_{c}\right)\right] .
\end{aligned}
$$

In the absence of low pass filters or with the filters omitted, the phase reactor and the converter transformer can be lumped together [13], eliminating the dependence on the complex filter bus voltage in (1) - (4). Similarly, if transformerless designs

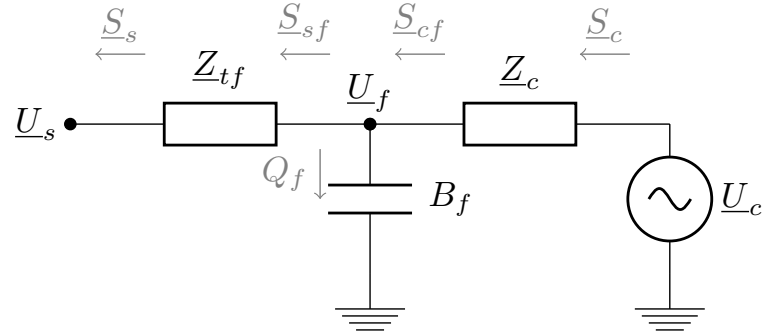

Fig. 2. Equivalent single phase power flow model of a converter station connected to the AC grid.

are considered, the filter bus and the AC grid bus coincide, hence simplifying the equations.

A VSC HVDC converter can independently control the active and reactive power injection with respect to the AC system. In the power flow algorithm presented in this paper, the active power injection is modeled in two different ways:

1) Constant $P$ : The converter has a constant active power injection $P_{s}$ into the AC grid.

2) Constant $U_{d c}$ : The algorithm adapts the active power injection $P_{s}$ to obtain a constant DC bus voltage $U_{d c}$.

The actual steady-state behavior of a two-terminal VSC HVDC link can be modeled in a power flow algorithm by one constant $P$ converter and one constant $U_{d c}$ converter. The active power injection of the latter is unknown prior to the power flow and depend on the losses in the VSC HVDC system. This constant $U_{d c}$ converter is referred to as the DC slack converter, due to its similarity to the slack bus in AC power flow algorithms.

This two-terminal representation can be extended to model $x$ different MTDC systems with a total of $y$ DC converters. With one DC slack bus per MTDC system, this results in $y-x$ converters controlling their active power output (constant $P$ ). The $x$ remaining DC slack converters, one per MTDC system, adjust their power to account for the DC system losses and to keep up the voltage of the DC grids. Alternatively, a voltage droop can be implemented to study the effect of distributing the DC voltage control function on the power flows [15].

The ability of the converter to independently control the reactive power results in two different representations in the power flow algorithm:

1) Constant $Q$ : The converter has a constant reactive power injection $Q_{s}$ into the AC grid.

2) Constant $U$ : The converter adapts the reactive power injection to obtain a constant $\mathrm{AC}$ bus voltage magnitude $U_{s}$.

As discussed in [8], commercial software models include the converter up to the filter bus while the filter and transformer have to be added separately. The power and voltage set-points are then defined with respect to the filter bus or the converter bus [11] instead of the system bus. Although these alternative methods are easier to implement, they do not comply with current day practices in VSC HVDC systems.

The model developed in this paper also includes the converter station losses using a generalized loss formula with 


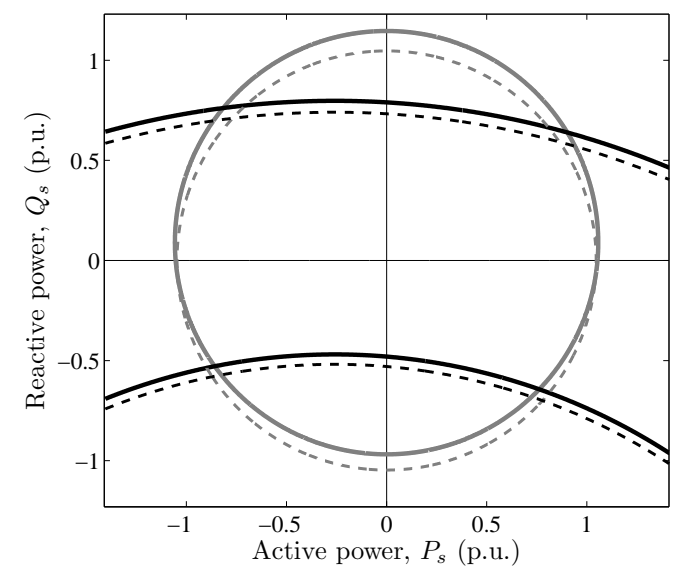

Fig. 3. PQ-capability chart - Converter station current and voltage limits (with $I_{c_{\max }}=1.05$ p.u., $U_{c_{\min }}=0.85$ p.u. and $U_{c_{\max }}=1.2$ p.u.).

the converter losses quadratically dependent on the converter current $I_{c}[16]$ :

$$
P_{\text {loss }}=a+b \cdot I_{c}+c \cdot I_{c}^{2},
$$

with the per unit converter current magnitude $I_{c}$ given by

$$
I_{c}=\frac{\sqrt{P_{c}^{2}+Q_{c}^{2}}}{\sqrt{3} U_{c}} .
$$

This aggregated loss model has been derived for the Södra Länken project, a VSC HVDC link with a rating of $600 \mathrm{MW}$ and a DC voltage of $\pm 300 \mathrm{kV}$. The model includes the losses in the different components of a VSC HVDC converter substation. The loss components are further discussed in [16]. The loss data in this paper has been scaled down to the appropriate MVA base.

\section{B. VSC HVDC Converter limits}

To ensure an overall safe operation of the converter station, the steady state working point must be situated within the PQ capability chart of the converter, depicted in Fig. 3. The full lines include the effect of the filters, the dotted lines neglect the filters. The gray circle forms the converter current limit. The black arcs depict the upper and lower converter voltage limits.

With the apparent power injection written as

$$
\underline{S}_{s}=\underline{U}_{s} \underline{I}_{s}^{*}
$$

the system bus voltage equation, which is equal to

$$
\underline{U}_{s}=\underline{U}_{f}-\underline{Z}_{t f} \underline{I}_{s},
$$

can be reformulated and substituted in the equation for the current at the filter bus, yielding

$$
\underline{I}_{s}=\underline{I}_{c}-\frac{\underline{U}_{s}+\underline{Z}_{t f} \underline{I}_{s}}{\underline{Z}_{f}} .
$$

Rewriting this equation in terms of the current $\underline{I}_{s}$ and substituting it in (12) results in a closed expression of the converter current limit

$$
\underline{S}_{s}=-U_{s}^{2}\left(\frac{1}{\underline{Z}_{f}^{*}+\underline{Z}_{t f}^{*}}\right)+\underline{U}_{s} \underline{I}_{c m}^{*}\left(\frac{\underline{Z}_{f}^{*}}{\underline{Z}_{f}^{*}+\underline{Z}_{t f}^{*}}\right),
$$

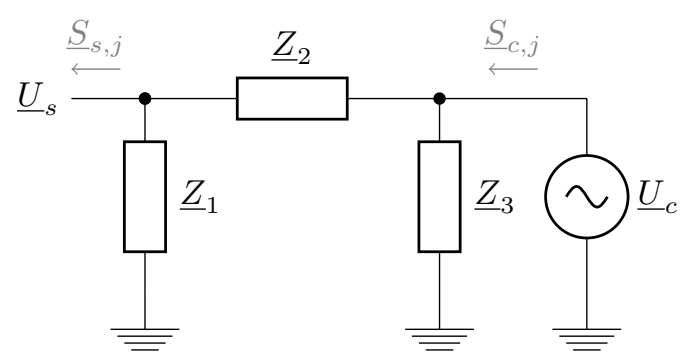

Fig. 4. Pi-equivalent scheme of a VSC HVDC converter.

with $\underline{I}_{c m}$ the phasor of the maximum current,

$$
\underline{I}_{c m}=I_{c_{\max }} \angle \delta_{I_{c}} \text {. }
$$

With the filters omitted, (15) simplifies to

$$
\underline{S}_{s}=\underline{U}_{s} \underline{I}_{c m}^{*}
$$

As shown in Fig. 3, the circular current limit from (17) slightly scales and shifts to a new center point as a result of the filter inclusion. The filter capacitance thus leads to a small bias in Q-axis direction [17].

In VSC systems that use Pulse Width Modulation (PWM), the modulation factor has an upper and lower limit, respectively to avoid overmodulation and the reappearance of harmonics. In [18], the lower reactive power limit is a constant at -0.5 p.u. With the Modular Multilevel Converter (MMC) technology, the reactive power is limited by the current in the lagging region [19]. The lower voltage limit can thus be omitted in this case.

These limits are implemented as limits on the converter voltage. The voltage limits can be obtained by applying a $Y-\Delta$ transformation to the equivalent model from Fig. 2. This is shown in Fig. 4, with the equivalent impedances $\underline{Z}_{1}$, $\underline{Z}_{2}$ and $\underline{Z}_{3}$ respectively

$$
\begin{aligned}
& \underline{Z}_{1}=\frac{\underline{Z}_{t f} \underline{Z}_{c}+\underline{Z}_{c} \underline{Z}_{f}+\underline{Z}_{f} \underline{Z}_{t f}}{\underline{Z}_{c}}, \\
& \underline{Z}_{2}=\frac{\underline{Z}_{t f} \underline{Z}_{c}+\underline{Z}_{c} \underline{Z}_{f}+\underline{Z}_{f} \underline{Z}_{t f}}{\underline{Z}_{f}} . \\
& \underline{Z}_{3}=\frac{\underline{Z}_{t f} \underline{Z}_{c}+\underline{Z}_{c} \underline{Z}_{f}+\underline{Z}_{f} \underline{Z}_{t f}}{\underline{Z}_{t f}} .
\end{aligned}
$$

Rewriting $\underline{I}_{s}^{*}$ in (12) in terms of the currents $\underline{I}_{1}$ and $\underline{I}_{2}$, through $\underline{Z}_{1}$ and $\underline{Z}_{2}$ results in

$$
\begin{aligned}
\underline{S}_{s} & =\underline{U}_{s}\left(\underline{I}_{2}^{*}-\underline{I}_{1}^{*}\right), \\
& =\underline{U}_{s}\left(\frac{\underline{U}_{c}^{*}-\underline{U}_{s}^{*}}{\underline{Z}_{2}^{*}}-\frac{\underline{U}_{s}^{*}}{\underline{Z}_{1}^{*}}\right) .
\end{aligned}
$$

Rewriting this equation in terms of admittances results in the expression for $\underline{S}_{s}$ in terms of a converter voltage limit

$$
\underline{S}_{s}=-U_{s}^{2}\left(\underline{Y}_{1}^{*}+\underline{Y}_{2}^{*}\right)+\underline{U}_{s} \underline{U}_{c m}^{*} \underline{Y}_{2}^{*}
$$

with $\underline{U}_{c m}$ the phasor of the minimum or maximum converter voltage $U_{c_{\min }}$ or $U_{c_{\max }} . U_{c_{\max }}$ is the maximum voltage that can be generated by the converter while avoiding overmodulation. The lower voltage limit $U_{c_{m i n}}$ in Fig. 3 has been chosen to comply with the minimum reactive power limit from [18]. 
This limit can be omitted in a MMC or when no minimum reactive power limit is imposed on the converter.

Similar expressions can be found by applying l'Hôpital's rule to (18) - (20) for $\underline{Z}_{f} \rightarrow \infty$ or $\underline{Z}_{t f} \rightarrow 0$ when the filter or transformer are not included. The expression without the filter hence simplifies to

$$
\underline{S}_{s}=-U_{s}^{2}\left(\frac{1}{\underline{Z}_{t f}^{*}+\underline{Z}_{c}^{*}}\right)+\frac{\underline{U}_{s} \underline{U}_{c m}^{*}}{\underline{Z}_{t f}^{*}+\underline{Z}_{c}^{*}} .
$$

Similar to the current limit, the inclusion of the filter shifts the voltage limit circles from (17) to a new center point, while their radii slightly change.

\section{DC Grid model}

The power flows in the DC grid can be obtained in a way similar to a conventional AC power flow. The current injected at a DC node $i$ can be written as the current flowing to the other $n-1$ nodes in the network:

$$
I_{d c_{i}}=\sum_{\substack{j=1 \\ j \neq i}}^{n} Y_{d c_{i j}} \cdot\left(U_{d c_{i}}-U_{d c_{j}}\right),
$$

with $Y_{d c_{i j}}$ equal to $1 / R_{d c_{i j}}$.

Combining all currents injected in an $n$ bus DC network results in

$$
I_{d c}=\mathrm{Y}_{\mathrm{dc}} \boldsymbol{U}_{d c}
$$

with $\boldsymbol{I}_{\boldsymbol{d c}}=\left[I_{d c_{1}}, I_{d c_{2}} \ldots I_{d c_{k}}, 0 \ldots 0\right]^{T}$ the DC current vector with $n-k$ zero elements due to DC buses without a power injection and converter outages, $\boldsymbol{U}_{d c}=\left[U_{d c_{1}}, U_{d c_{2}} \ldots U_{d c_{n}}\right]^{T}$ the DC voltage vector and $Y_{d c}$ the DC bus matrix. When line outages are taken into account, the DC bus matrix needs to be updated accordingly. When $x$ DC grids are considered, all DC bus matrices $Y_{\mathrm{dc} j}$ of the different DC grids are combined in one single sparse band matrix $Y_{d c}$ :

$$
\mathrm{Y}_{\mathrm{dc}}=\left[\begin{array}{ccc}
\mathrm{Y}_{\mathrm{dc} 1} & & \\
& \ddots & \\
& & \mathrm{Y}_{\mathrm{dc} x}
\end{array}\right] \text {. }
$$

For a monopolar DC grid the active power injected in node $i$ can be written as

$$
P_{d c_{i}}=U_{d c_{i}} I_{d c_{i}}, \quad \forall i \leq k,
$$

whereas for a monopolar, symmetrically grounded DC grid, the power injections become

$$
P_{d c_{i}}=2 U_{d c_{i}} I_{d c_{i}}, \quad \forall i \leq k .
$$

A similar expressions holds for a bipolar configuration. The current injections $\boldsymbol{I}_{\boldsymbol{d c}}$ are not known prior to the power flow solution for the DC grid, whereas the active power injections $\boldsymbol{P}_{\boldsymbol{d c}}$ are known for all buses except for the DC slack buses as a results of the AC power flow. Combining (26) and (29) and assuming a monopolar, symmetrically grounded DC grid

$$
P_{d c_{i}}=2 U_{d c_{i}} \sum_{\substack{j=1 \\ j \neq i}}^{n} Y_{d c_{i j}} \cdot\left(U_{d c_{i}}-U_{d c_{j}}\right) .
$$

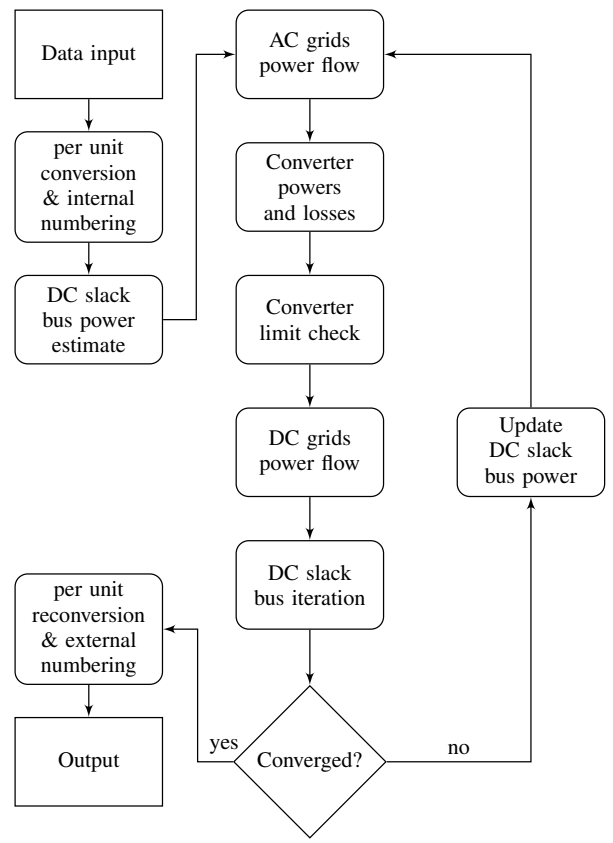

Fig. 5. Flow chart of the sequential VSC AC/DC power flow algorithm.

This system of non-linear equations can either be solved with a Newton-Raphson (NR) method, as derived in the next section, or rewritten to include the unknown DC slack buses power equations directly [20].

\section{AC/DC POWER FLOW}

Fig. 5 shows the flow chart of the sequential power flow algorithm. The algorithm starts with a per unit conversion and an internal bus renumbering from [8] which facilates the inclusion of multiple DC grids, line and converter outages, as well as DC buses without an AC grid connection. In order to simplify notation, it is assumed in the remainder of the paper that interconnected AC and DC buses have the same bus number. For convenience, the analysis will be confined to one AC grid and one DC grid with $n$ buses of which $k$ have a connection to the AC grids. The method can easily be extended to include multiple $\mathrm{AC}$ and $\mathrm{DC}$ grids.

\section{A. AC network power flow}

The power flow equations for bus $i$ in the AC grid can be written as

$$
\begin{aligned}
& P_{i}(\boldsymbol{U}, \boldsymbol{\delta})=U_{i} \sum_{j=1}^{p} U_{j}\left[G_{i j} \cos \left(\delta_{i}-\delta_{j}\right)+B_{i j} \sin \left(\delta_{i}-\delta_{j}\right)\right], \\
& Q_{i}(\boldsymbol{U}, \boldsymbol{\delta})=U_{i} \sum_{j=1}^{p} U_{j}\left[G_{i j} \sin \left(\delta_{i}-\delta_{j}\right)-B_{i j} \cos \left(\delta_{i}-\delta_{j}\right)\right] .
\end{aligned}
$$

The non-linear set of power flow equations for all $\mathrm{AC}$ buses can be solved using a NR power flow, with the converter power injections $\boldsymbol{P}_{\boldsymbol{s}}$ and $\boldsymbol{Q}_{\boldsymbol{s}}$ included in the power mismatch vectors $\boldsymbol{\Delta} \boldsymbol{P}^{(k)}$ and $\boldsymbol{\Delta} \boldsymbol{Q}^{(k)}$ as negative loads. The power mismatch vectors can be rewritten as:

$$
\begin{aligned}
& \Delta P_{i}^{(j)}=P_{i}^{g e n}-\left(P_{i}^{\text {dem }}-P_{s_{i}}\right)-P_{i}\left(\boldsymbol{U}^{(j)}, \boldsymbol{\delta}^{(j)}\right), \\
& \Delta Q_{i}^{(j)}=Q_{i}^{\text {gen }}-\left(Q_{i}^{d e m}-Q_{s_{i}}\right)-Q_{i}\left(\boldsymbol{U}^{(j)}, \boldsymbol{\delta}^{(j)}\right) .
\end{aligned}
$$


Converters in $V$-control are represented as dummy AC generators and their $\mathrm{AC}$ buses are changed from $P Q$-nodes to $P V$-nodes. The active power injections of the slack buses are changed in order to control the DC grid voltages. Without lack of generality, the first converter of the DC grid is assumed to operate as DC slack bus to simplify notation. As a first estimate to initiate the iteration, the DC system is assumed to be lossless, hence

$$
P_{s_{1}}^{(0)}=-\sum_{j=2}^{k} P_{s_{j}} .
$$

For subsequent iterations, the solution from the previous iteration is used for $P_{s_{1}}^{(k)}$.

\section{B. Converter calculations}

With the AC grid voltages $\underline{U}_{s}$ and power injection at the AC grid side $\underline{S}_{s}$ known, the converter side voltage and currents can be calculated. With (12) - (13), the filter bus voltage $\underline{U}_{f}$ can be written as

$$
\underline{U}_{f_{i}}=\underline{U}_{s_{i}}+\underline{Z}_{t f_{i}} \cdot\left(\underline{S}_{s_{i}}^{*}\right) \quad \forall i<k .
$$

The converter current $\underline{I}_{c}$ can be extracted from (14), with $\underline{I}_{s}$ rewritten using (12)

$$
\underline{I}_{c_{i}}=\frac{\underline{U}_{s_{i}}}{\underline{Z}_{f_{i}}}+\left(\frac{\underline{S}_{s_{i}}^{*}}{\underline{U}_{s_{i}}^{*}}\right) \cdot \frac{\underline{Z}_{f_{i}}+\underline{Z}_{t f_{i}}}{\underline{Z}_{f_{i}}} \forall i<k
$$

which can be substituted in (10) to calculate the converter losses. The converter voltage $\underline{U}_{c}$ can be calculated as

$$
\underline{U}_{c_{i}}=\underline{U}_{f_{i}}+\underline{Z}_{c_{i}} \underline{I}_{c_{i}}, \quad \forall i<k .
$$

With all quantities on the AC side known, the DC grid's injected power becomes

$$
P_{d c_{i}}=-P_{c, i}-P_{l o s s_{i}}, \quad \forall i<k .
$$

\section{Converter limit check}

If the converter is operating outside its limits, the power injections are adapted to ensure a safe operation. In general, a weighting factor can be used to give a higher priority to active or reactive power. In this paper, priority is given to active power. The grid injected power $P_{s}$ can remain unaltered if intersections with the capability chart can be found. If, on the contrary, $P_{s}$ is too large, the active power is limited to its maximum value and the reactive power is adapted accordingly.

The current and voltage limits in (15) and (23) are circles in the $P Q$-plane. Hence, with $P_{s}$ known, the two reactive power limits imposed by the current limit can be calculated as

$$
Q_{s}=Q_{0} \pm \sqrt{r^{2}-\left(P_{s}-P_{0}\right)^{2}},
$$

with the circle's center $\underline{S}_{0}=P_{0}+\jmath Q_{0}$ and a radius $r$. The center point and radius of the current limit are given by

$$
\begin{gathered}
\underline{S}_{0}=-U_{s}^{2}\left(\frac{1}{\underline{Z}_{f}^{*}+\underline{Z}_{t f}^{*}}\right), \\
r=U_{s} I_{c_{\max }}\left|\frac{\underline{Z}_{f}^{*}}{\underline{Z}_{f}^{*}+\underline{Z}_{t f}^{*}}\right| .
\end{gathered}
$$

The two solutions refer to the upper and lower reactive power limit imposed by the current limit.

Similarly, the voltage limits center point and radius are given by

$$
\begin{aligned}
\underline{S}_{0} & =-U_{s}^{2}\left(\underline{Y}_{1}^{*}+\underline{Y}_{2}^{*}\right), \\
r & =U_{s} U_{c m} Y_{2} .
\end{aligned}
$$

Replacing $U_{c m}$ in (44) with $U_{c_{\min }}$ or $U_{c_{\max }}$ and substituting (43) - (44) in (40) gives an analytical expression for the lower and upper voltage limit reactive power. Only the solution with the positive sign is of interest, as the other solution refers to the lower crossing point with voltage limit circle.

The limit checks can be implemented similarly to those of generator limits: If a converter is operating outside its active power limits, the set-points must be reduced to their maximum values. Additionally, it must be ascertained that the DC slack buses are working within their bounds. Apart from the active power violations, the converter's reactive power must stay within limits. When a $Q$-limit is hit, the most stringent reactive power limit imposes the new converter operation point. If the converter is operating in $V$-control, the voltage controlled bus also has to be changed to a $P Q$-bus. If one or more converters hit their limits, the most stringent one is enforced and the convergence flag is reset.

Contrary to generator limits, which are often approximated by constant values, the $Q$-limits of the converters are updated every iteration due to changing grid conditions. As a consequence, it was found that simply enforcing the limits during each iteration proved unsatisfactory. Therefore, the set-points are only updated when the incremental change in apparent power exceeds a minimum value of 1e-2 p.u. Alternatively, it was also found that checking the limits after every iteration and only updating their value every three iterations led to convergence as well. The algorithm should be properly tuned to achieve good convergence.

\section{DC network power flow}

Alternatively to the approach proposed in [20], the nonlinear DC network equations from (30) can be solved with a NR method

$$
\left(\boldsymbol{U}_{\boldsymbol{d c}} \frac{\partial \boldsymbol{P}_{\boldsymbol{d c}}}{\partial \boldsymbol{U}_{\boldsymbol{d c}}}\right)^{(j)} \cdot \frac{\boldsymbol{\Delta} \boldsymbol{U}_{\boldsymbol{d c}}}{\boldsymbol{U}_{\boldsymbol{d c}}}{ }^{(j)}=\boldsymbol{\Delta} \boldsymbol{P}_{\boldsymbol{d c}}{ }^{(j)} .
$$

The power mismatch vector $\boldsymbol{\Delta} \boldsymbol{P}_{\boldsymbol{d c}}{ }^{(j)}$ is given by

$$
\Delta P_{d c_{i}}^{(j)}=\left\{\begin{array}{cc}
P_{d c_{i}}^{(k)}-P_{d c_{i}}\left(\boldsymbol{U}_{\boldsymbol{d c}}{ }^{(j)}\right) & \forall i \leq k \\
-P_{d c_{i}}\left(\boldsymbol{U}_{\boldsymbol{d c}}{ }^{(j)}\right) & \forall k \leq i \leq n
\end{array},\right.
$$

with superscripts $(j)$ and $(k)$ respectively referring to the inner NR iteration and the outer AC/DC power flow iteration. The terms of the Jacobian are

$$
\begin{aligned}
& \left(U_{d c_{j}} \frac{\partial P_{d c_{i}}}{\partial U_{d c_{j}}}\right)^{(j)}=-2 U_{d c_{i}}^{(j)} Y_{d c_{i j}} U_{d c_{j}}^{(j)}, \\
& \left(U_{d c_{i}} \frac{\partial P_{d c_{i}}}{\partial U_{d c_{i}}}\right)^{(j)}=P_{d c_{i}}^{(j)}+2 U_{d c_{i}}^{(j)^{2}} \sum_{\substack{j=1 \\
j \neq i}}^{n} Y_{d c_{i j}} .
\end{aligned}
$$


The equations and terms corresponding to the slack bus are removed since its voltage is known prior to the DC network power flow. After convergence, the voltages on all DC buses are known, while the slack bus power injection can be found using (30). The DC line currents can be obtained by premultiplying the DC bus voltage vector $\boldsymbol{U}_{d c}$ by the system branch admittance matrices $Y_{d c f}$ and $Y_{d c t}$, which are obtained as a byproduct of the construction of the DC bus matrix $Y_{d c}$.

\section{E. DC slack bus iteration}

The AC bus active power injection $P_{s_{1}}$ of the DC slack bus is calculated from its DC power $P_{d c_{1}}$ by accounting for the converter losses. As the converter losses from (10) depend on the yet unknown converter current, an additional iteration is needed to calculate the active power injection $P_{s_{1}}$. During this iteration, the grid side voltage $\underline{U}_{S_{1}}$ and reactive power injection $Q_{s_{1}}$ are kept constant. Omitting the subscript 1 to simplify notations, the value of $P_{c}$ is iteratively updated according to

$$
P_{c}^{(i)}=-P_{d c}^{(k)}-P_{l o s s}^{(i)},
$$

with the superscripts $(i)$ and $(k)$ respectively referring to the DC slack bus iteration and the outer AC/DC power flow iteration. The converter losses $P_{\text {loss }}$ are calculated from (10) - (11). The previous AC network power flow results are used to provide an initial estimate for the converter losses $P_{\text {loss }}^{(0)}$.

A NR iteration based on $\underline{U}_{c}$ and $\underline{U}_{f}$ as variables is internally used to update the converter state in order to obtain a new value for $P_{\text {loss }}^{(i)}$, as shown in (50) at the bottom of the page, with the functions $F_{1}$ and $F_{2}$ given by

$$
\begin{aligned}
& F_{1}\left(\underline{U}_{s}, \underline{U}_{c}, \underline{U}_{f}\right)=P_{c f}-P_{s f}, \\
& F_{2}\left(\underline{U}_{s}, \underline{U}_{c}, \underline{U}_{f}\right)=Q_{c f}-Q_{s f}-Q_{f} .
\end{aligned}
$$

The power mismatches $\Delta P_{c}^{(j)}$ and $\Delta Q_{s}^{(j)}$ can be calculated by respectively using (3) and (2) and are given by

$$
\begin{aligned}
& \Delta P_{c}^{(j)}=P_{c}^{(i)}-P_{c}\left(\underline{U}_{f}^{(j)}, \underline{U}_{c}^{(j)}\right), \\
& \Delta Q_{s}^{(j)}=Q_{s}^{(k)}-Q_{s}\left(\underline{U}_{s}^{(k)}, \underline{U}_{f}^{(j)}\right),
\end{aligned}
$$

The superscript $(j)$ refers to the inner NR iteration. The elements of the Jacobian matrix can be analytically derived from (1) - (9) directly and by substitution in the expressions for $F_{1}$ and $F_{2}$. The results are given in the appendix.

In absence of the transformer, the active and reactive power injections can be rewritten in terms of $\underline{U}_{s}$ and $\underline{U}_{c}$ as

$$
\begin{aligned}
& P_{s}=-U_{s}^{2} G_{c}+U_{s} U_{c}\left[G_{c} \cos \left(\delta_{s}-\delta_{c}\right)+B_{c} \sin \left(\delta_{s}-\delta_{c}\right)\right],(55) \\
& Q_{s}=U_{s}^{2} B_{c f}+U_{s} U_{c}\left[G_{c} \sin \left(\delta_{s}-\delta_{c}\right)-B_{c} \cos \left(\delta_{s}-\delta_{c}\right)\right],(56)
\end{aligned}
$$

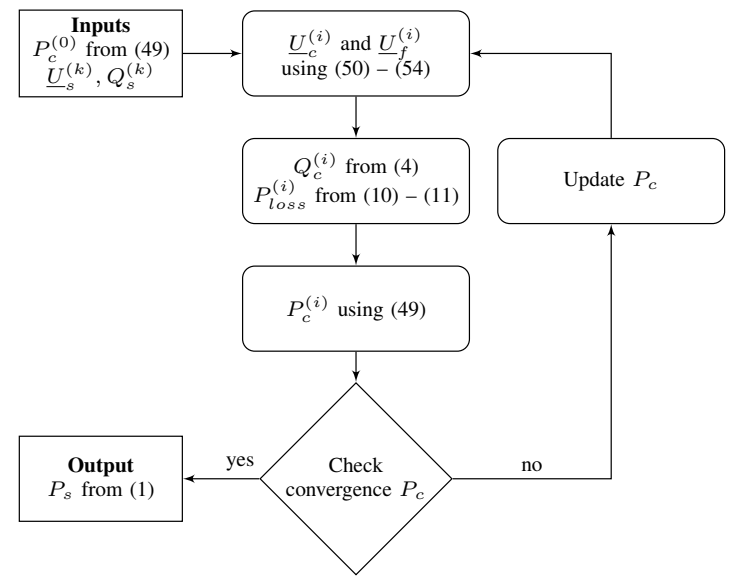

Fig. 6. DC Slack bus iteration flow chart.

with $B_{c}+B_{f}$ abbreviated as $B_{c f}$. By rewriting $Q_{s}$, the Jacobian matrix simplifies to a $2 \times 2$ structure retaining $\Delta \delta_{c}$ and $\frac{\Delta U_{c}}{U_{c}}$ as unknowns and the derivates related to the $P_{c}$ and $Q_{s}$ equations as Jacobian elements

$$
\left[\begin{array}{ll}
\left(\frac{\partial P_{c}}{\partial \delta_{c}}\right)^{(j)} & \left(U_{c} \frac{\partial P_{c}}{\partial U_{c}}\right)^{(j)} \\
\left(\frac{\partial Q_{s}}{\partial \delta_{c}}\right)^{(j)} & \left(U_{c} \frac{\partial Q_{s}}{\partial U_{c}}\right)^{(j)}
\end{array}\right]\left[\begin{array}{c}
\Delta \delta_{c}^{(j)} \\
\frac{\Delta U_{c}}{U_{c}}(j)
\end{array}\right]=\left[\begin{array}{c}
\Delta P_{c}^{(j)} \\
\Delta Q_{s}^{(j)}
\end{array}\right]
$$

with $\left(\frac{\partial P_{c}}{\partial \delta_{c}}\right)^{(j)}$ and $\left(U_{c} \frac{\partial P_{c}}{\partial U_{c}}\right)^{(j)}$ given by the expressions in the appendix and the other elements of the reduced Jacobian matrix given by

$$
\begin{aligned}
& \left(\frac{\partial Q_{s}}{\partial \delta_{c}}\right)^{(j)}=-P_{s}^{(j)}-U_{s}^{(k)}{ }^{2} G_{c}, \\
& \left(U_{c} \frac{\partial Q_{s}}{\partial U_{c}}\right)^{(j)}=Q_{s}^{(j)}-U_{s}^{(k)^{2}} B_{c f},
\end{aligned}
$$

with $P_{s}, Q_{s}$ from (55) - (56) and $P_{c}, Q_{c}$ from (3) - (4) with $\underline{U}_{f}$ replaced by $\underline{U}_{s}$. In absence of the converter filters, the equations can be reduced to the forms derived in [13].

When multiple DC grids and slack buses are present, all Jacobian matrices are combined and solved simultaneously.

\section{Simulation RESUlts}

This section presents simulation results of the proposed AC/DC power flow algorithm implemented in the open-source Matlab toolbox, MATPOWER [14]. In the case of commercial closed-source AC power flow software, the model has to be integrated with the coding level and not at the end-user level, hence making the implementation less evident.

$$
\left[\begin{array}{cccc}
\left(\frac{\partial P_{c}}{\partial \delta_{c}}\right)^{(j)} & \left(\frac{\partial P_{c}}{\partial \delta_{f}}\right)^{(j)} & \left(U_{c} \frac{\partial P_{c}}{\partial U_{c}}\right)^{(j)} & \left(U_{f} \frac{\partial P_{c}}{\partial U_{f}}\right)^{(j)} \\
0 & \left(\frac{\partial Q_{s}}{\partial \delta_{f}}\right)^{(j)} & 0 & \left(U_{f} \frac{\partial Q_{s}}{\partial U_{f}}\right)^{(j)} \\
\left(\frac{\partial F_{1}}{\partial \delta_{c}}\right)^{(j)} & \left(\frac{\partial F_{1}}{\partial \delta_{f}}\right)^{(j)} & \left(U_{c} \frac{\partial F_{1}}{\partial U_{c}}\right)^{(j)} & \left(U_{f} \frac{\partial F_{1}}{\partial U_{f}}\right)^{(j)} \\
\left(\frac{\partial F_{2}}{\partial \delta_{c}}\right)^{(j)} & \left(\frac{\partial F_{2}}{\partial \delta_{f}}\right)^{(j)} & \left(U_{c} \frac{\partial F_{2}}{\partial U_{c}}\right)^{(j)} & \left(U_{f} \frac{\partial F_{2}}{\partial U_{f}}\right)^{(j)}
\end{array}\right] \cdot\left[\begin{array}{c}
\Delta \delta_{c}^{(j)} \\
\Delta \delta_{f}^{(j)} \\
\frac{\Delta U_{c}(j)}{U_{c}}(j) \\
\frac{\Delta U_{f}}{U_{f}}
\end{array}\right]=\left[\begin{array}{c}
\Delta P_{c}^{(j)} \\
\Delta Q_{s}^{(j)} \\
-F_{1}^{(j)} \\
-F_{2}^{(j)}
\end{array}\right]
$$




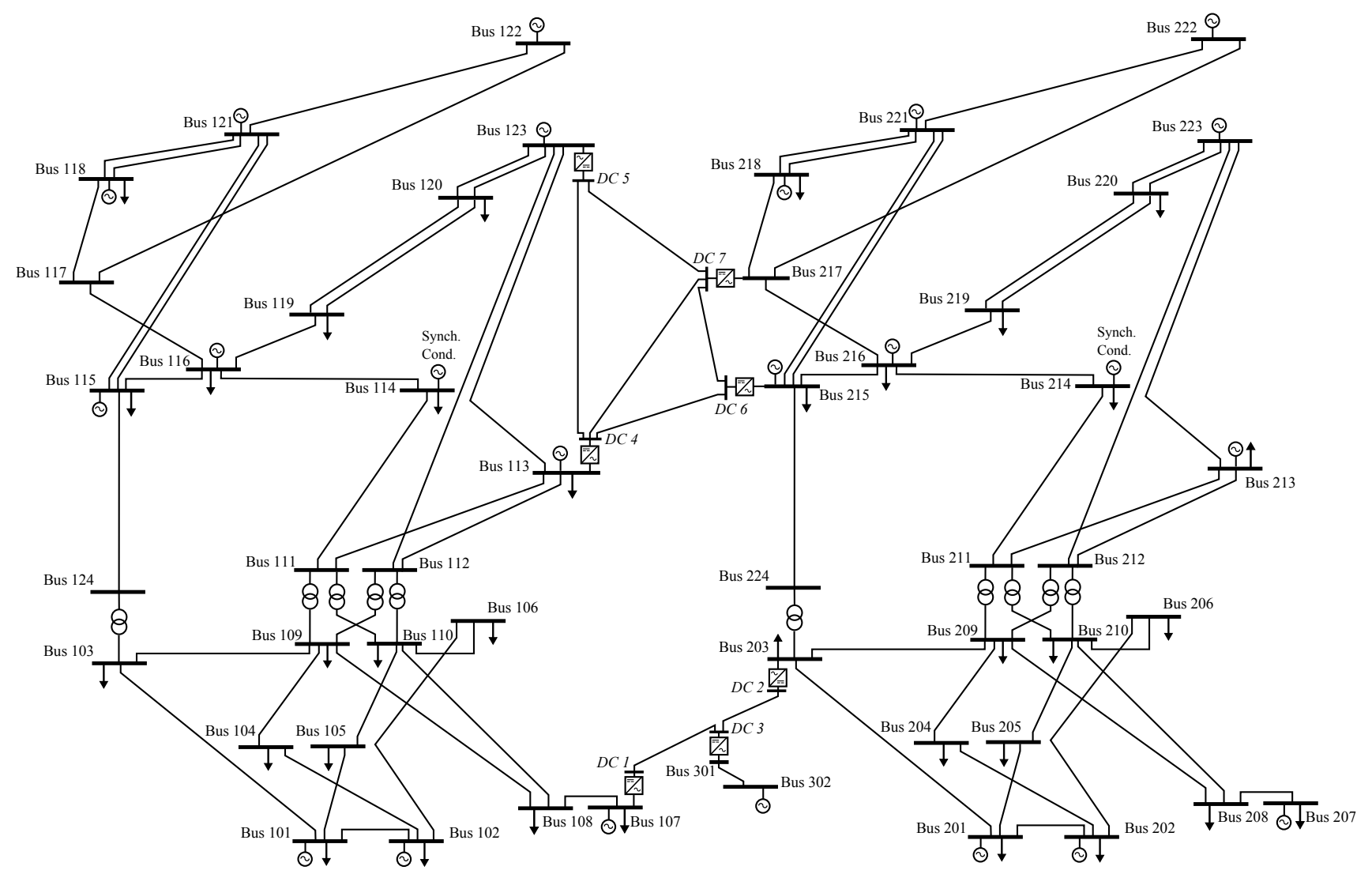

Fig. 7. Modified Two Area RTS-96 system with 2 MTDC Systems.

TABLE I

VSC CONVERTER DATA

\begin{tabular}{l|c||c|cccc}
\hline \multicolumn{1}{l||}{} \\
\multicolumn{1}{l||}{ Converter parameters } & \multicolumn{5}{c}{ Rating \& Converter loss data } \\
\hline \hline$X_{t r}($ p.u. $)$ & 0.1121 & $P_{d c}(M W)$ & 100 & 200 & 200 & 100 \\
$R_{t r}$ (p.u.) & 0.0015 & $\pm V_{d c}(k V)$ & 150 & 150 & 300 & 300 \\
\cline { 3 - 7 }$B_{f}$ (p.u.) & $0.0887^{\mathrm{a}}$ & $a(M W)$ & 1.103 & 2.206 & 1.103 & 2.206 \\
$X_{c}$ (р.u.) & 0.16428 & $b(k V)$ & 0.887 & 0.887 & 1.800 & 1.800 \\
$R_{c}$ (p.u.) & 0.0001 & $c_{r e c}(\Omega)$ & 2.885 & 1.442 & 5.94 & 11.88 \\
& & $c_{\text {inv }}(\Omega)$ & 4.371 & 2.185 & 9 & 18 \\
\hline
\end{tabular}

${ }^{a}$ Only included for converters $4-7$.

In this implementation, a tolerance of $1 \mathrm{e}-8$ p.u. was used for the AC and DC networks power flow, DC slack bus iteration and the overall iteration loop. Results are presented on a modified version of the IEEE Two Area RTS-96 (MRTS) network [21]. A single-line diagram of the MRTS network is shown in Fig. 7. In this MRTS network, the three interconnections between the two areas have been replaced by MTDC systems: In the $138 \mathrm{kV}$ system, line $107-203$ has been replaced by a 3-terminal $150 \mathrm{kV}$ MTDC system connecting the two asynchronous systems with a $150 \mathrm{MW}$ offshore wind farm (buses 301 and 302). In the $345 \mathrm{kV}$ system, lines $113-215$ and $123-217$ have been replaced by a 4-terminal $300 \mathrm{kV}$ MTDC system. The parameters of the DC systems can be found in Tab. I. The 3-terminal DC system between buses 107 and 203 represents a filterless MMC VSC MTDC system, the 4-terminal MTDC scheme includes low-pass filters and
TABLE II

POWER FLOW SOLUTION - MTDC GRID 1

\begin{tabular}{c|ccc}
\hline Converter & 1 & 2 & 3 \\
\hline \hline Control & Slack - Q & $\mathrm{P}-\mathrm{V}$ & $\mathrm{P}-\mathrm{Q}$ \\
$P_{s}(M W)$ & 66.84 & 75.00 & -150.00 \\
$Q_{s}(M V A r)$ & 0.00 & 5.86 & 0.00 \\
$U_{s}(p . u)$. & 1.025 & 1.000 & 1.050 \\
$\delta_{s}($ deg $)$ & -8.93 & -4.88 & -0.08 \\
$P_{c}(M W)$ & 66.91 & 75.09 & -149.67 \\
$Q_{c}(M V A r)$ & 11.75 & 21.50 & 56.40 \\
$U_{c}(p . u)$. & 1.042 & 1.038 & 1.120 \\
$\delta_{c}($ deg $)$ & 1.04 & 6.63 & -20.73 \\
$P_{l o s s}(M W)$ & 1.56 & 1.67 & 3.52 \\
\hline$P_{d c}(M W)$ & -68.47 & -76.76 & 146.16 \\
$U_{d c}($ p.u. $)$ & 1.000 & 0.999 & 1.006 \\
\hline \multicolumn{4}{|c}{}
\end{tabular}

represents a 2-level PWM VSC MTDC system. The three sequentially solved AC networks operate asynchronously, with reference (slack) buses at 113, 213 and 302. A generator (U100), producing $80 \mathrm{MW}$, at bus 107 and a generator (U76), producing $76 \mathrm{MW}$, at bus 201 have been disabled and replaced by the production of $150 \mathrm{MW}$ in bus 302 . The active power injections of the 4-terminal MTDC grid converters closely resemble the line flows between the different zones in the original two-area MRTS network. All but one converters are set to reactive power control, working at unity power factor. Tabs. II - III summarize the AC and DC powers and voltages of the VSC converters connected to the 2 MTDC grids.

The power flow converges in 2 overall iterations with a 
TABLE III

POWER FLOW SOLUTION - MTDC GRID 2

\begin{tabular}{c|cccc}
\hline Converter & 4 & 5 & 6 & 7 \\
\hline \hline Control & Slack - Q & $\mathrm{P}-\mathrm{Q}$ & $\mathrm{P}-\mathrm{Q}$ & $\mathrm{P}-\mathrm{Q}$ \\
$P_{s}(M W)$ & 124.43 & -50.00 & -135.00 & 50.00 \\
$Q_{s}(M V A r)$ & 0.00 & 0.00 & 0.00 & 0.00 \\
$U_{s}($ p.u. $)$ & 1.020 & 1.050 & 1.014 & 1.039 \\
$\delta_{s}($ deg $)$ & 0.00 & 10.11 & 10.25 & 14.60 \\
$P_{c}(M W)$ & 124.67 & -49.96 & -134.72 & 50.04 \\
$Q_{c}(M V A r)$ & 31.36 & -3.46 & 39.26 & -3.14 \\
$U_{c}($ p.u. $)$ & 1.061 & 1.042 & 1.062 & 1.033 \\
$\delta_{c}($ deg $)$ & 18.41 & 2.90 & -9.89 & 21.96 \\
$P_{l o s s}(M W)$ & 2.81 & 1.36 & 3.04 & 1.33 \\
\hline$P_{d c}(M W)$ & -127.49 & 48.60 & 131.67 & -51.37 \\
$U_{d c}(p . u)$. & 1.000 & 1.008 & 1.010 & 1.006 \\
\hline
\end{tabular}

flat start. In the first overall iteration cycle, all grids need 4 internal iteration steps. In the second overall cycle, the AC grid connected to the DC slack buses is the only grid that needs 3 steps to converge, the other grids are already converged after the first cycle. Compared to the situation without MTDC systems, the calculation time increases with a factor 2.4 , with the largest contributions resulting from the repeated $\mathrm{AC}$ power flow $(75 \%)$ and the DC slack iteration (21\%). The DC power flow is only responsible for $1.7 \%$ of the overall calculation time due to the limited size of the DC grids compared to the AC grids. Since no converter limits are encountered, enabling the limits does not increase the overall number of iterations. However, the overall calculation time increases by a factor 1.3.

\section{CONCLUSION}

In this paper, a steady-state VSC MTDC model for power flow programs has been developed. The model allows simulating multiple AC grids interconnected by multiple DC grids. The approach demonstrated in this paper is general and allows to include converter limits as well as different converter topologies by either including converter transformers and/or low-pass filters. Simulation results show the ability of the proposed model to fully represent MTDC systems in power flow software and demonstrate how the model can be incorporated with an open-source $\mathrm{AC}$ power flow analysis code.

\section{APPENDIX}

The slack bus iteration Jacobian elements from (50) are

$$
\begin{aligned}
& \left(\frac{\partial P_{c}}{\partial \delta_{c}}\right)^{(j)}=-Q_{c}^{(j)}-U_{c}^{(j)}{ }^{2} B_{c}, \\
& \left(\frac{\partial P_{c}}{\partial \delta_{f}}\right)^{(j)}=Q_{c}^{(j)}+U_{c}^{(j)}{ }^{2} B_{c}, \\
& \left(U_{c} \frac{\partial P_{c}}{\partial U_{c}}\right)^{(j)}=P_{c}^{(j)}+U_{c}^{(j)}{ }^{2} G_{c}, \\
& \left(U_{f} \frac{\partial P_{c}}{\partial U_{f}}\right)^{(j)}=P_{c}^{(j)}-U_{c}^{(j)}{ }^{2} G_{c}, \\
& \left(\frac{\partial Q_{s}}{\partial \delta_{f}}\right)^{(j)}=-P_{s}^{(j)}-U_{s}^{(k)}{ }^{2} G_{t f},
\end{aligned}
$$

$$
\begin{aligned}
& \left(U_{f} \frac{\partial Q_{s}}{\partial U_{f}}\right)^{(j)}=Q_{s}^{(j)}-U_{s}^{(k)^{2}} B_{t f}, \\
& \left(\frac{\partial F_{1}}{\partial \delta_{c}}\right)^{(j)}=Q_{c f}^{(j)}-U_{f}^{(j)}{ }^{2} B_{c}, \\
& \left(\frac{\partial F_{1}}{\partial \delta_{f}}\right)^{(j)}=-Q_{c f}^{(j)}+Q_{s f}^{(j)}+U_{f}^{(j)^{2}}\left(B_{c}+B_{t f}\right), \\
& \left(U_{c} \frac{\partial F_{1}}{\partial U_{c}}\right)^{(j)}=P_{c f}^{(j)}+{U_{f}^{(j)}}^{2} G_{c}, \\
& \left(U_{f} \frac{\partial F_{1}}{\partial U_{f}}\right)^{(j)}=P_{c f}^{(j)}-P_{s f}^{(j)}-U_{f}^{(j)^{2}}\left(G_{c}+G_{t f}\right), \\
& \left(\frac{\partial F_{2}}{\partial \delta_{c}}\right)^{(j)}=-P_{c f}^{(j)}-U_{f}^{(j)} G_{c}, \\
& \left(\frac{\partial F_{2}}{\partial \delta_{f}}\right)^{(j)}=P_{c f}^{(j)}-P_{s f}^{(j)}+U_{f}^{(j)}{ }^{2}\left(G_{c}+G_{t f}\right), \\
& \left(U_{c} \frac{\partial F_{2}}{\partial U_{c}}\right)^{(j)}=Q_{c f}^{(j)}-U_{f}^{(j)}{ }^{2} B_{c}, \\
& \left(U_{f} \frac{\partial F_{2}}{\partial U_{f}}\right)^{(j)}=Q_{c f}^{(j)}-Q_{s f}^{(j)}+{U_{f}^{(j)}}^{2}\left(B_{c}+B_{t f}+2 B_{f}\right),
\end{aligned}
$$

with the converter powers from (1) - (9).

\section{REFERENCES}

[1] D. Van Hertem and M. Ghandhari, "Multi-terminal VSC HVDC for the European supergrid: Obstacles," Renewable and Sustainable Energy Reviews, vol. 14, no. 9, pp. 3156-3163, Dec. 2010.

[2] W. Lu and B.-T. Ooi, "Optimal acquisition and aggregation of offshore wind power by multiterminal voltage-source HVDC," IEEE Trans. Power Del., vol. 18, no. 1, pp. 201-206, Jan. 2003.

[3] C. M. Ong and A. Hamzei-nejad, "A general-purpose multiterminal DC load-flow," IEEE Trans. Power App. Syst., vol. PAS-100, no. 7, pp. 31663174, Jul. 1981.

[4] H. Fudeh and C. M. Ong, "A simple and efficient AC-DC load-flow method for multiterminal DC systems," IEEE Trans. Power App. Syst., vol. PAS-100, no. 11, pp. 4389-4396, Nov. 1981.

[5] T. Smed, G. Andersson, G. B. Sheble, and L. L. Grigsby, "A new approach to AC/DC power flow," IEEE Trans. Power Syst., vol. 6, no. 3, pp. 1238-1244, Aug. 1991.

[6] M. El-Marsafawy and R. Mathur, "A new, fast technique for loadflow solution of integrated multi-terminal AC/DC systems," IEEE Trans. Power App. Syst., vol. 99, no. 1, pp. 246-255, Jan./Feb. 1980.

[7] M. E. El-Hawary and S. T. Ibrahim, "A new approach to AC-DC load flow analysis," Electric Power Systems Research, vol. 33, no. 3, pp. 193-200, 1995.

[8] J. Beerten, S. Cole, and R. Belmans, "Implementation aspects of a sequential AC/DC power flow computation algorithm for multi-terminal VSC HVDC systems," in Proc. IET ACDC '10, London, UK, Oct. 2021, 2010.

[9] C. Angeles-Camacho, O. L. Tortelli, E. Acha, and C. R. Fuerte-Esquivel, "Inclusion of a high voltage DC-voltage source converter model in a Newton-Raphson power flow algorithm," IEE Proc.-Gener. Transm. and Distrib., vol. 150, no. 6, pp. 691-696, Nov. 2003.

[10] A. Pizano-Martinez, C. R. Fuerte-Esquivel, H. Ambriz-Perez, and E. Acha, "Modeling of VSC-based HVDC systems for a NewtonRaphson OPF algorithm," IEEE Trans. Power Syst., vol. 22, no. 4, pp. 1794-1803, Nov. 2007.

[11] G. Li, M. Zhou, J. He, G. Li, and H. Liang, "Power flow calculation of power systems incorporating VSC-HVDC," in Proc.IEEE PowerCon 2004, vol. 2, Singapore, Nov. 21-24, 2004, pp. 1562-1566.

[12] M. Zhao, Z. Chen, and F. Blaabjerg, "Load flow analysis for variable speed offshore wind farms," IET Renew. Power Gener, vol. 3, no. 2, pp. 120-132, Jun. 2009.

[13] J. Beerten, S. Cole, and R. Belmans, "A sequential AC/DC power flow algorithm for neworks containing multi-terminal VSC HVDC systems," in Proc. IEEE PES GM '10, Minneapolis, USA, Jul. 25-29, 2010. 
[14] R. D. Zimmerman, C. E. Murillo-Sanchez, and R. J. Thomas, "MATPOWER: Steady-state operations, planning, and analysis tools for power systems research and education," IEEE Trans. Power Syst., vol. 26, no. 1, pp. 12-19, Feb. 2011

[15] J. Beerten, D. Van Hertem, and R. Belmans, "VSC MTDC systems with a distributed DC voltage control - a power flow approach," in Proc. IEEE PowerTech '11, Trondheim, Norway, Jun. 19-23, 2011.

[16] G. Daelemans, "VSC HVDC in meshed networks," Master's thesis, Katholieke Universiteit Leuven, Leuven, 2008

[17] S. Johansson, G. Asplund, E. Jansson, and R. Rudervall, "Power system stability benefits with VSC DC-transmission systems," in Proc. CIGRE 2004 Session, Paris, France, 2004.

[18] M. P. Bahrman and B. K. Johnson, "The ABCs of HVDC transmission technologies," IEEE Power Energy Mag., vol. 5, no. 2, pp. 32-44, 2007.

[19] G. P. Adam, O. Anaya-Lara, and G. Burt, "Steady-state and transient performance of DC transmission systems based on HVDC technology," in Proc. IET ACDC '10, London, UK, Oct. 20-21, 2010.

[20] S. Cole, J. Beerten, and R. Belmans, "Generalized dynamic VSC MTDC model for power system stability studies," IEEE Trans. Power Syst., vol. 25, no. 3, pp. 1655-1662, Aug. 2010.

[21] Reliability Test System Task Force, "The IEEE reliability test system1996. a report prepared by the reliability test system task force of the application of probability methods subcommittee," IEEE Trans. Power Syst., vol. 14, no. 3, pp. 1010-1020, Aug. 1999.

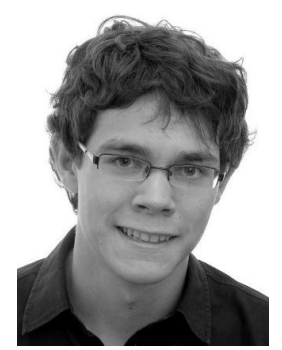

Jef Beerten (S'07) was born in Belgium in 1985. $\mathrm{He}$ received the M.Sc. degree in electrical engineering from the Katholieke Universiteit Leuven (K.U.Leuven), Leuven, Belgium, in 2008, where he is currently working towards the Ph.D. degree. He is a Research Assistant with the division ESATELECTA division of K.U.Leuven. His research interests include power system control, the grid of the future and multiterminal VSC HVDC in particular. Mr. Beerten is an active member of both the IEEE and Cigré.

Mr. Beerten holds a Ph.D. fellowship from the Research Foundation Flanders (FWO).

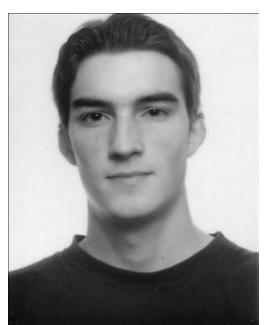

Stijn Cole (S'06-M'10) received the M.Sc. degree in electrical engineering in 2005 from the Katholieke Universiteit Leuven (K.U.Leuven), Belgium. After completing his doctoral dissertation on modelling and simulation of HVDC systems, he received the $\mathrm{Ph} . \mathrm{D}$. degree in 2010 from the same university. In 2010, he joined Tractebel Engineering where he is currently working as a power system consultant.

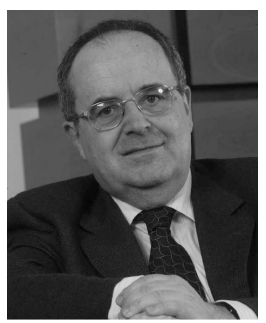

Ronnie Belmans (S'77-M'84-SM'89-F'05) received the M.Sc. degree in electrical engineering in 1979 and the Ph.D. degree in 1984, both from the K.U.Leuven, Belgium, the Special Doctorate in 1989 and the Habilitierung in 1993, both from the RWTH, Aachen, Germany. Currently, he is a full professor with the K.U.Leuven, teaching electric power and energy systems. His research interests include techno-economic aspects of power systems, power quality and distributed generation. He is also guest and Technology, London-UK. professor at Imperial College of Science, Medicine professor 